\title{
Cosmic-Ray Elemental Spectra Measured with ISS-CREAM
}

R. Takeishi ${ }^{* 1}$, Y. Amare ${ }^{2}$, D. Angelaszek ${ }^{2,3}$, N. Anthony ${ }^{2}$, G. H. Choi ${ }^{1}$, M. Chung ${ }^{2}$, M. Copley², L. Derome ${ }^{4}$, L. Eraud ${ }^{4}$, C. Falana ${ }^{2}$, A. Gerrety ${ }^{2}$, L. Hagenau ${ }^{2}$, J. H. Han², H. G. Huh ${ }^{2}$, Y. S. Hwang ${ }^{5, \dagger}$, H. J. Hyun ${ }^{5, \ddagger}$, H. B. Jeon ${ }^{5}$, J. A. Jeon ${ }^{1, \S}$, S. Jeong ${ }^{1}$, S. C. Kang ${ }^{5}$, H. J. Kim ${ }^{5}$, K. C. Kim ${ }^{2}$, M. H. Kim ${ }^{2}$, H. Y. Lee ${ }^{1, \S}$, J. Lee ${ }^{1,5}$, M. H. Lee ${ }^{2, \S}$, C. Lamb ${ }^{2}$, J. Liang ${ }^{2}$, L. Lu ${ }^{2}$, J. P. Lundquist ${ }^{1,2}$, L. Lutz ${ }^{2}$, B. Mark ${ }^{2}$, A. Menchaca-Rocha ${ }^{6}$, T. Mernik ${ }^{2}$, M. Nester ${ }^{2}$, O. Ofoha ${ }^{2}$, H. Park ${ }^{5}$, I. H. Park ${ }^{1}$, J. M. Park ${ }^{5}$ ฯ, N. Picot-Clemente ${ }^{2}$, S. Rostsky ${ }^{2}$, E. S. Seo ${ }^{2,3}$, J. R. Smith ${ }^{2, \|, ~ T . ~ T a t o l i ~}{ }^{2}$, P. Walpole ${ }^{2}$, R. P. Weinmann ${ }^{2}$, J. $\mathbf{W u}^{2}$, Z. Yin ${ }^{2,3}$, Y. S. Yoon ${ }^{2,3, * *}$ and H. G. Zhang ${ }^{2}$ for the ISS-CREAM Collaboration ${ }^{\dagger \dagger}$

${ }^{1}$ Dept. of Physics, Sungkyunkwan University, Suwon, Republic of Korea

${ }^{2}$ Inst. for Phys. Sci. and Tech., University of Maryland, College Park, MD, USA

${ }^{3}$ Dept. of Physics, University of Maryland, College Park, MD, USA

${ }^{4}$ Laboratoire de Physique Subatomique et de Cosmologie, Grenoble, France

${ }^{5}$ Dept. of Physics, Kyungpook National University, Daegu, Republic of Korea

${ }^{6}$ Instituto de Fisica, Universidad Nacional Autonoma de Mexico, Mexico City, Mexico

E-mail: takeishieskku.edu

The Cosmic Ray Energetics And Mass experiment for the International Space Station (ISSCREAM) is a direct cosmic-ray detection experiment deployed on the ISS in August 2017. It aims to reveal the sources, acceleration processes, and propagation of cosmic rays by observing individual elemental spectra at energies in the TeV-PeV range. ISS-CREAM consists of multiple complementary particle detectors. This work utilizes the Silicon Charge Detector (SCD) to measure cosmic-ray charges from protons to iron nuclei with a resolution of $0.1-0.3 \mathrm{e}$, and the calorimeter (CAL) to determine the cosmic-ray track and measure its energy by sampling the shower energy deposit of secondary particles. With more than 1-year of observations, we analyzed cosmic-ray spectra of various prominent species such as protons, helium, carbon and oxygen nuclei. We will report preliminary elemental spectra of cosmic rays for energies greater than about $10 \mathrm{TeV}$.

36th International Cosmic Ray Conference -ICRC2019-

July 24th - August 1st, 2019

Madison, WI, U.S.A.

\footnotetext{
* Speaker.

${ }^{\dagger}$ Currently at Korea Multi-purpose Accelerator Complex, Korea Atomic Energy Research Institute, Kyungju, Republic of Korea

$\ddagger$ Currently at Pohang Accelerator Laboratory, Pohang, Republic of Korea

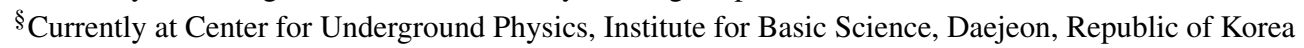

ICurrently at Advanced Radiation Technology Institute, Korea Atomic Energy Research Inst., Jeongeup, Republic of Korea

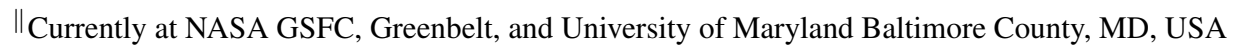

${ }^{* *}$ Currently at Korea Research Institute of Standards and Science, Daejeon, Republic of Korea

${ }^{\dagger \dagger}$ for collaboration list see PoS(ICRC2019)1177
} 


\section{Introduction}

The origin of cosmic rays and how they propagate are long-standing mysteries. The main components of the cosmic ray energy spectra have power-law shapes. Cosmic rays with energies greater than $10^{14} \mathrm{eV}$ have been detected via ground-based observations of air showers, which are secondary particle cascades induced by primary cosmic rays in the atmosphere. These measurements have shown that the all-particle spectrum has a feature known as the "knee" corresponding to a spectral steepening at $\sim 10^{15} \mathrm{eV}$. This could be explained by the acceleration limit of galactic cosmic rays generated by shock acceleration in supernova remnants.

Ground-based measurements provide a large collecting power, but they cannot unambiguously identify the primary particle type that initiated the shower because the air shower development has large fluctuations. Direct measurements with satellite or balloon-borne detectors can more precisely identify the primary particle type and determine its energy. Precise measurements of the energy dependence of elemental spectra from $\sim 10^{12} \mathrm{eV}$ to $\sim 10^{15} \mathrm{eV}$, where the expected rigiditydependent supernova acceleration limit could be reflected in a composition change, provide a key to understanding cosmic-ray acceleration and propagation mechanisms.

The balloon-borne Cosmic Ray Energies And Mass (CREAM) experiment was flown over Antarctica between 2004 and 2010, obtaining 161 days of flight time. The analysis of CREAM data showed that $\mathrm{TeV}$ energy spectra are harder than the lower energy data from previous experiments [1, 2]. A hardening of spectra of various nuclei has also been reported by PAMELA [3], AMS-02 [4, 5, 6, 7] and CALET [8]. The exact cause of spectral hardening is under investigation, while there are possible explanations that derive the hardening from acceleration mechanisms or nearby sources.

The Cosmic Ray Energetics And Mass experiment for the International Space Station (ISSCREAM) experiment aims to reveal the origin, acceleration and propagation mechanisms of cosmic rays with energies up to $10^{15} \mathrm{eV}$ by direct measurement. It utilizes a CREAM payload installed on the ISS and obtained more statistics the CREAM balloon experiments. We will report preliminary elemental spectra of cosmic rays with more than 1 year of ISS-CREAM observation time.

\section{ISS-CREAM Experiment}

The ISS-CREAM payload was launched on August 14, 2017 and placed on the ISS. Scientific observations started on August 22, 2017. The ISS-CREAM instrument is configured with complementary charge and energy measurement systems [9]. It consists of a Silicon Charge Detector (SCD), calorimeter (CAL), Top/Bottom Counting Detectors (TCD/BCD) and Boronated Scintillator Detector (BSD).

The SCD consists of four layers of silicon pixel sensors, placed at the top of the ISS-CREAM payload [10]. The active area in each layer is $78.2 \mathrm{~cm} \times 73.6 \mathrm{~cm}$ and segmented into $1.55 \mathrm{~cm}$ $\times 1.38 \mathrm{~cm}$ pixels to minimize hits of accompanying backscattered particles in the same segment as the incident particle. Each layer has 2,688 channels and therefore the SCD a total of 10,752 channels. Silicon pixel sensors are fabricated on 525- $\mu \mathrm{m}$ thick wafers with 16 pixels in a $4 \times 4$ array. Each sensor is attached to a flexible printed circuit board for signal readout inter-connection. Seven silicon sensors form a ladder, which is a basic electrical unit powered on/off and monitored. 
Various voltage, current and temperature sensors are installed and monitored in each ladder. Analog electronics boards are equipped with the CR1.4 application specific integrated circuit (ASIC) chips [11]. Each CR1.4 chip has 16 channels, and each channel has a preamplifier followed by a shaper. When each chip receives a trigger signal, it holds the voltages of the shaped signals at the trigger time for all 16 channels, and multiplexes the voltages into an output signal. Digital electronics boards are located off the active area to avoid adding material in the path of charged particles. They consist of Analog to Digital Converter (ADC) chips, Field Programmable Gate Array (FPGA) chips and voltage regulators. Each board carries out digitization of multiplexed analog signals.

The CAL has same basic design with the calorimeter used in the CREAM experiment [12]. It consists of a carbon target, 20 tungsten layers, and scintillating fiber ribbons. The tungsten and ribbon components are $50 \mathrm{~cm} \times 50 \mathrm{~cm}$ in area and about $10 \mathrm{~cm}$ in height. The tungsten layers, which have 20 radiation lengths in total, are interleaved with ribbon layers oriented alternately in the $\mathrm{x}$ - and $\mathrm{y}$-directions. Each layer consists of 50 fiber ribbons to measure the development of cosmic-ray showers. The light signal from each ribbon is collected and split into three sub-bundles with different gains to cover a wide dynamic range, and lead to a hybrid photodiode (HPD) through a bundle of clear fibers. Output signals of the HPD are sent to an ADC chip located on ASIC boards.

The TCD and BCD each consist of a plastic scintillator and 400 photodiodes. The TCD is located between the carbon target and the $\mathrm{CAL}$, and the $\mathrm{BCD}$ is located below the CAL. The active areas of the T/BCD are $50 \mathrm{~cm} \times 50 \mathrm{~cm}$ and $60 \mathrm{~cm} \times 60 \mathrm{~cm}$, respectively. They provide shower profiles for electron/proton separation and an additional lower energy threshold trigger for the CAL. The BSD consists of a $60 \mathrm{~cm} \times 60 \mathrm{~cm} \times 3.8 \mathrm{~cm}$ boron-loaded plastic scintillator. It measures the late-time ( $\sim$ several $\mu \mathrm{s})$ scintillation light and neutron activity produced by cosmic-ray induced showers. The BSD enhances the electron/hadron discrimination power using the fact that hadroninduced showers generate more late scintillation light and neutrons than those originating from electrons, compared in the same cosmic ray energy.

The ISS-CREAM trigger for science events are provided by two instruments. The CAL trigger covers higher energy events and is created when at least six consecutive layer ribbons detect energy deposits larger than the threshold value, which is $\sim 40 \mathrm{MeV}$ and determined in each ribbon, and at least one of them shows energy deposit larger than $76 \mathrm{MeV}$. The T/BCD trigger is created when one channel in TCD and two channels in BCD show larger energy deposits than threshold values determined in each channel. The trigger efficiency is studied by calculating the fraction of events satisfying the trigger condition among all events which traverse the SCD active area and the bottom of the CAL, using Geant3 Monte-Carlo (MC) simulations. The preliminary value is about 70$80 \%$ for events which have incident energies above $\sim 4 \mathrm{TeV}$. Details of the ISS-CREAM MC are explained elsewhere [13].

\section{Analysis}

\subsection{Event Selection and Tracking}

For this analysis, we use ISS-CREAM data recorded from August 22, 2017, through February 12, 2019. The events triggered by CAL and T/BCD are used. Reconstruction of the shower track 
is based on the method used in the CREAM balloon analysis [2] 14]. In each event, the hit position of the shower axis in each CAL layer is determined using ribbons with the highest energy deposit and the neighboring two ribbons in the layer. The incident particle track is reconstructed from a linear fit of the hit positions in the $X Z$ and $Y Z$ planes. We required $\chi^{2} / n d f$ values less than 10 for the tracks. If distances from a hit position to the neighboring layer hit positions are larger than a threshold value, the position are excluded from the fit as a misidentified position. Figure 1 shows an example CAL event tracking.

\subsection{Charge Determination}

To determine the incident particle charge, the reconstructed shower axis from the CAL is extrapolated to the SCD layer. We use the SCD layer 1 for this analysis since the charge change between layers is under further investigation. Then a fixed pixel area centered on the extrapolated position is searched to obtain the highest pixel signal. The searching area is optimized to sustain more than $90 \%$ charge identification efficiency in energy bins greater than several $\mathrm{TeV}$ and determined to be $7 \times 7$ pixels in area, as shown in Figure 2 The highest pixel signal is corrected for the path length in the sensor calculated from the reconstructed incident direction.

The SCD signal is proportional to the square of the incident charge $\left(Z^{2}\right)$ since it is due to the ionization energy loss. To calculate the incident charge, we use conversion relations from SCD signals to the charge, determined from following methods. For $Z<5$, we adopt SCD beam test data performed on the ground [10]. The charge distribution using the CAL tracking and the SCD signal search explained above is shown in Figure 3 (left). For $Z \geq 5$, we use another conversion relation including a correction factor determined from a tracking fit utilizing the hits on the three active SCD layers for T/BCD trigger events. This is because peak positions of the measured charge distribution calculated using the beam test data has a systematic shift from CREAM balloon results for

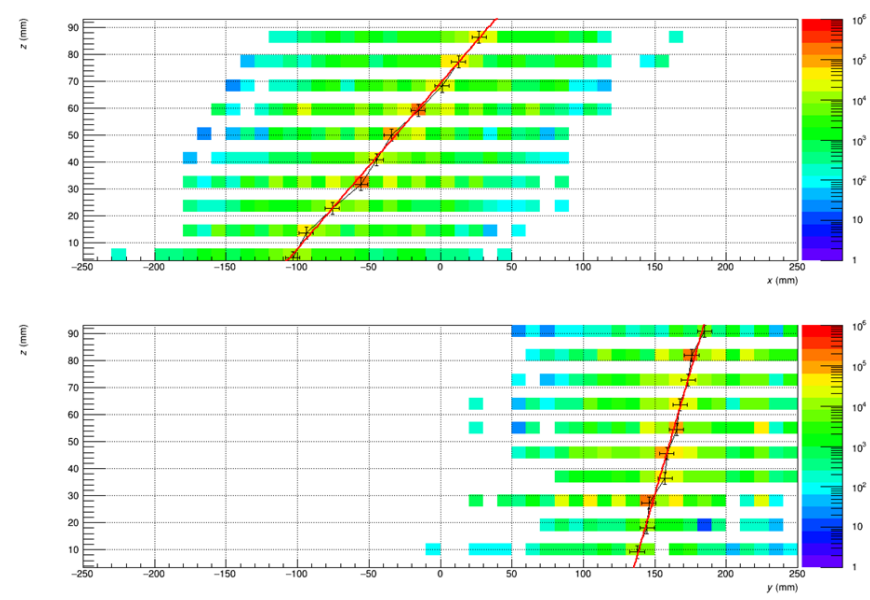

Figure 1: An example event of the CAL tracking. The color shows the energy deposit (MeV unit) in each ribbon. The cross represents hit positions in each CAL layer determined from the method in the text. The red lines show reconstructed tracks projected onto $X Z$ and $Y Z$ planes. The sum of the energy deposits is $2751 \mathrm{GeV}$. The incident particle energy is reconstructed as $2008 \mathrm{TeV}$. Note that the energy calibration is preliminary. (top) $X Z$ plane. (bottom) $Y Z$ plane. 

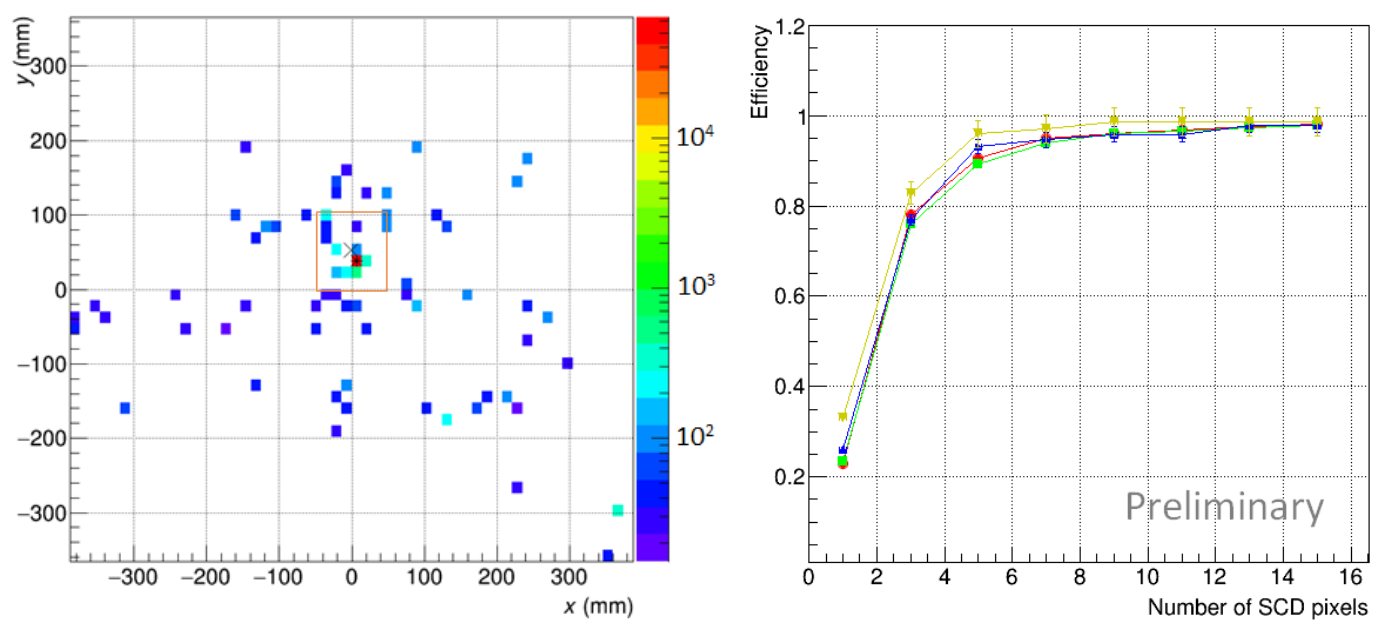

Figure 2: (left) An example SCD event. The color corresponds to the ADC value. The $\mathrm{X}$ mark is the extrapolated position of the reconstructed track from the CAL. The orange square indicates the $7 \times 7$ pixel search area for the maximum signal. In this example, the maximum signal is the red pixel indicated by the asterisk. (right) SCD selection efficiency for different search area sizes using proton MC. The red, green, blue, yellow describe incident energy $10^{3.6} \mathrm{GeV}-10^{3.8} \mathrm{GeV}, 10^{3.8} \mathrm{GeV}-10^{4.0} \mathrm{GeV}, 10^{4.0} \mathrm{GeV}-10^{4.2} \mathrm{GeV}$, $10^{4.2} \mathrm{GeV}-10^{4.4} \mathrm{GeV}$, respectively. With a search area of $7 \times 7$ pixels, the efficiency is more than $90 \%$. The effect of SCD noisy and dead pixels, which are about $10 \%$ on average, is not included in the calculation.

$Z \gtrsim 5$, and it needs higher statistics to clarify the correction factor. We picked up all combinations of SCD hits between the three layers, and selected events which shows charge consistency within \pm 1 . We also fit the three hits by a linear line and required a $\chi^{2} / n d f$ value less than 10 . Contamination of background particles are negligible since they are mostly small signals corresponding to protons or helium nuclei. Then the correction factor of the shift is determined by comparing charge distribution of selected events with CREAM balloon results. The resulting SCD charge distribution is shown in Figure 3 (right).

Events with $0.7<Z<1.7$ are selected as protons, while events with $1.7<Z<2.7$ are selected as helium nuclei. Also, events with $(\mathrm{n}-0.5)<Z<(\mathrm{n}+0.5)$ are selected as charge $\mathrm{n}$ for heavier nuclei. The charge resolutions are estimated to be $0.1-0.3 \mathrm{e}$ for the most prominent species, which are protons, helium, carbon, oxygen, neon, magnesium, silicon and iron nuclei. Noisy SCD channels identified by their large root-mean-square pedestal variations were excluded from the analysis. Throughout the analysis data period, $\sim 10 \%$ of the 2688 SCD channels are masked, including dead and noisy channels.

\subsection{Energy Measurement}

The CAL is designed to sample the energy deposit from showers initiated by cosmic rays with energies up to $\sim 10^{15} \mathrm{eV}$. Conversion factors from ADC signals to energy deposits are obtained from the ratio of the energy of the MC deposited in each ribbon to the ADC signal measured at calibration beam tests performed on the ground. The tests are using electron and pion beams with energies from tens of $\mathrm{GeV}$ to hundreds of $\mathrm{GeV}$. Energy deposits in $\mathrm{CAL}$ ribbons and energy resolution in 

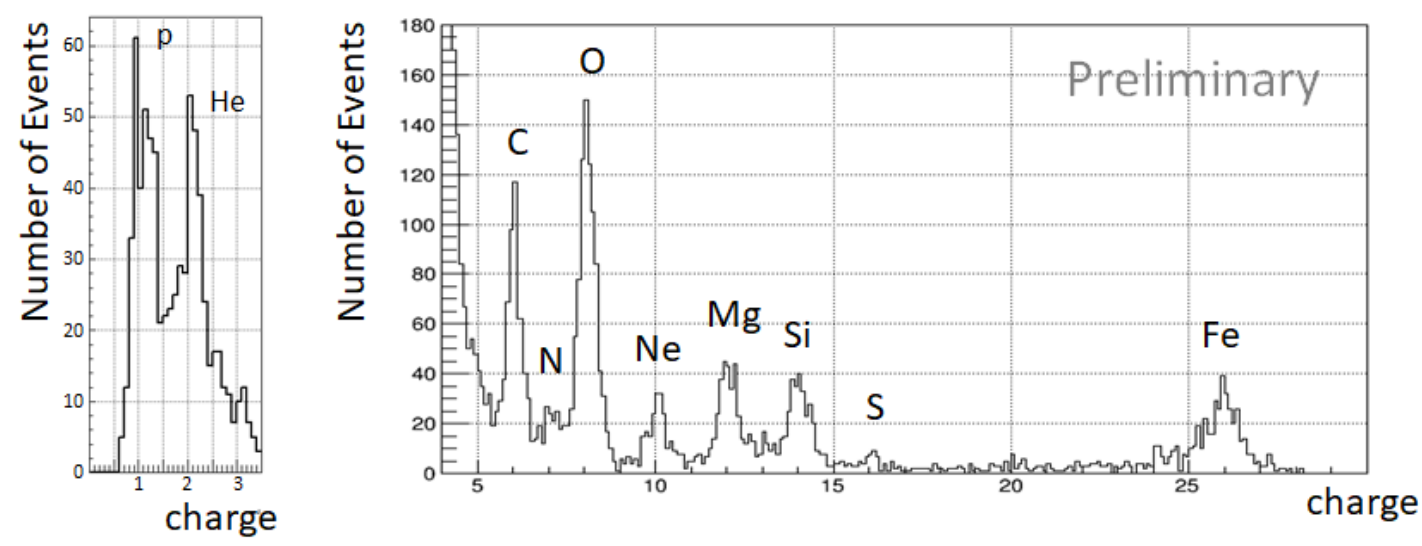

Figure 3: Charge distributions of sampled data. (left) Proton and helium components obtained from the CAL tracking and SCD signal search. (right) Species for larger charge components obtained from the SCD tracking.

each incident energy are obtained, and compared with previous beam tests for CREAM balloon flights. Details of the CAL energy calibration are explained elsewhere [15, 16, 17]. Each ribbon in the CAL has three energy ranges with different gains. The inter-calibration between the ranges are carried out with flight data by comparing signals from different ranges of the same ribbon generated by the same artificial input signal. In this analysis, the low gain range signals are used. The higher ranges will be used in the next analysis, which can make obtained particle energies larger. The incident particle energy is determined from the sum of energy deposits in the CAL using the conversion relation found from MC [13]. Note that the calibration of the ISS-CREAM CAL energy measurement is preliminary since the channel-by-channel correction is not done completely.

\subsection{Spectrum Calculation}

The measured spectra need to be corrected for the instrument acceptance and the live time of the observation. In this analysis, we calculated $d N / d E$, which is not corrected flux by the acceptance and the live time, where $d N$ is the number of events in an energy bin, $d E$ is the energy bin size. The estimation of the acceptance and live time effects are in progress.

\section{Results}

Figure 4 shows the measured differential spectra with energies larger than $10 \mathrm{TeV}$ for each element. The spectra are set as an arbitrary unit. The left figure shows elemental particle spectra for protons and helium nuclei. The maximum energy of the proton flux reaches about $1 \mathrm{PeV}$. The spectra follow power-law shapes with indexes close to 2.5-2.7, but data points are fluctuated more than statistical errors since the analysis is premature. To solve this, we will include spectrum deconvolution [14] and background subtraction in each charge and energy for the next analysis step. Various efficiencies will have to be studied to extend the measurements to lower energies. Since efficiencies are different in each energy and charge, we will include energies less than 10 

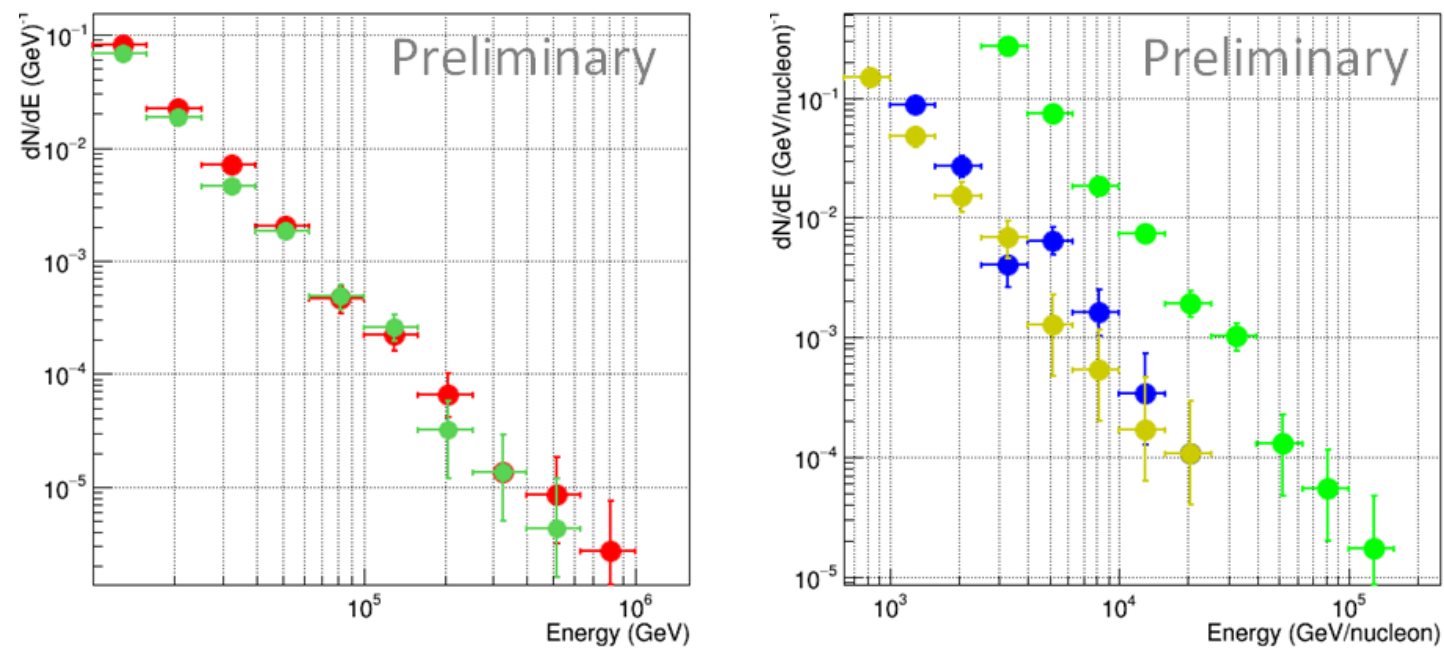

Figure 4: Preliminary elemental particle spectra measured with ISS-CREAM. The vertical error bars represent statistical errors. Since larger energy bins are used at higher energies, we divide the number of events by the bin width to follow $d E=1 \mathrm{GeV}$, so the vertical values can be less than unity. (left) Light nuclei. The red and green represent protons and helium nuclei, respectively. (right) Heavy nuclei in energy per nucleon unit. The green, blue and yellow represent helium, carbon and oxygen nuclei, respectively.

$\mathrm{TeV}$ after careful studies are done. The right figure shows elemental particle spectra for helium, carbon and oxygen nuclei as functions of energy per nucleon. These spectra also follow powerlaw shapes. The relative abundance has no physical significance because necessary corrections for interactions and propagation have not been applied to these data yet. The carbon might be enhanced by charge misidentified events from protons and helium nuclei, since the background subtraction is not completed yet. These effects will also be corrected after spectrum deconvolution and background studies are done.

\section{Summary}

We presented the preliminary methods and results of cosmic-ray spectra measured with ISSCREAM. The preliminary spectra showed reasonable power-law shapes. Current analysis does not include the detector acceptance and the observation live time for absolute fluxes. Also, flux deconvolution and background estimation are necessary for precise determination of the fluxes. Studies of such effects in each energy and charge are underway, and we plan to complete them and publish the results soon.

\section{Acknowledgements}

This work was supported in the U.S. by NASA grant NNX17AB41G, in Korea by National Research Foundation grants 2018R1A2A1A05022685 and 2018R1A6A1A06024970, and their predecessor grants. It was also supported in France by IN2P3/CNRS and CNES and in Mexico by DGAPA-UNAM project IN109617. The authors thank NASA GSFC WFF and its contractors for 
engineering support and project management, JSC ISS Program Office for the launch support and ISS accommodation, MSFC for the operational support, and KSC and SpaceX for the launch support.

\section{References}

[1] H. S. Ahn et al., Discrepant hardening observed in cosmic-ray elemental spectra, Astrophys. J. Lett. 714 (2010) L89-L92.

[2] Y. S. Yoon et al., Proton and Helium Spectra from the CREAM-III Flight, Astrophys. J. 839 (2017) 5.

[3] O. Adriani et al., PAMELA measurements of cosmic-ray proton and helium spectra, Science 332 (2011) 69-76.

[4] M. Aguilar et al. (AMS Collaboration), Precision Measurement of the Proton Flux in Primary Cosmic Rays from Rigidity $1 \mathrm{GV}$ to $1.8 \mathrm{TV}$ with the Alpha Magnetic Spectrometer on the International Space Station, Phys. Rev. Lett. 114 (2015) 171103.

[5] M. Aguilar et al. (AMS Collaboration), Precision Measurement of the Helium Flux in Primary Cosmic Rays of Rigidities 1.9 GV to 3 TV with the Alpha Magnetic Spectrometer on the International Space Station, Phys. Rev. Lett. 115 (2015) 211101.

[6] M. Aguilar et al. (AMS Collaboration), Observation of the Identical Rigidity Dependence of He, C, and $O$ Cosmic Rays at High Rigidities by the Alpha Magnetic Spectrometer on the International Space Station, Phys. Rev. Lett. 119 (2017) 251101.

[7] M. Aguilar et al. (AMS Collaboration), Observation of New Properties of Secondary Cosmic Rays Lithium, Beryllium, and Boron by the Alpha Magnetic Spectrometer on the International Space Station, Phys. Rev. Lett. 120 (2018) 021101.

[8] O. Adriani et al. (CALET Collaboration), Direct Measurement of the Cosmic-Ray Proton Spectrum from $50 \mathrm{GeV}$ to $10 \mathrm{TeV}$ with the Calorimetric Electron Telescope on the International Space Station, Phys. Rev. Lett. 122 (2019) 181102.

[9] E. S. Seo et al., Cosmic Ray Energetics And Mass for the International Space Station (ISS-CREAM), Adv. Space Res. 53 (2014) 1451-1455.

[10] J. Lee et al., The ISS-CREAM Silicon Charge Detector for identification of the charge of cosmic rays up to $Z=26$ : Design, fabrication and ground-test performance, Astropart. Phys. 112 (2019) 8-15.

[11] J. H. Adams et al., The CR-1 chip: custom VLSI circuity for cosmic rays, Proc. 26th ICRC 5 (1999) 69-71.

[12] M. H. Lee et al., Performance of a CREAM-III calorimeter, IEEE Trans. Nucl. Sci. 56 (2009) 1396-1399.

[13] J. Wu et al., Monte Carlo Simulations of the ISS-CREAM Instrument, submitted to PoS (ICRC2019).

[14] Y. S. Yoon et al., Cosmic-ray Proton and Helium Spectra from the First CREAM Flight, Astrophys. J. 728 (2011) 122.

[15] N. Picot-Clemente et al., Performance of the ISS-CREAM Calorimeter, Proc. 35th ICRC (2017) 247.

[16] J. H. Han et al., Calibration of the CREAM calorimeter with beam test data, Proc. 32th ICRC 6 (2011) 392.

[17] H. S. Ahn et al., Performance of CREAM Calorimeter: Results of Beam Tests, Nucl. Phys. B (Poc. Suppl.) 150 (2006) 272-275. 